\title{
Reprogramming A375 cells to induced-resembled neuronal cells by structured overexpression of specific transcription genes
}

\author{
HENGZHU ZHANG ${ }^{*}$, MIN WEI*, YANGYANG JIANG ${ }^{*}$, XIAODONG WANG, LEI SHE, \\ ZHENGCUN YAN, LUN DONG, LUJUN PANG and XINGDONG WANG
}

\author{
Department of Neurosurgery, Northern Jiangsu People's Hospital, Yangzhou, Jiangsu 225001, P.R. China
}

Received June 25, 2015; Accepted July 6, 2016

DOI: $10.3892 / \mathrm{mmr} .2016 .5598$

\begin{abstract}
Induced-resembled neuronal cells (irNCs) are generated by reprogramming human melanoma cells through the introduction of key transcription factors, providing novel concepts in the treatment of malignant tumor cells and making it possible to supply neural cells for laboratory use. In the present study, irNCs were derived from A 375 cells by inducing the 'forced' overexpression of specific genes, including achaete-scute homolog 1 (Ascl1), neuronal differentiation factor 1 (Neurod1), myelin transcription factor 1 (Myt1), brain protein 2 (Brn2, also termed POU3F2) and human brain-derived neurotrophic factor (h-BDNF). irNCs induced from A375 cells express multiple neuronal markers and fire action potentials, exhibiting properties similar to those of motor neurons. The reprogramming procedure comprised reverse transcription-polymerase chain reaction and immunofluorescence staining; furthermore, electrophysiological profiling demonstrated the characteristics of the induced-resembled neurons. The present study obtained a novel type of human irNC from human melanoma, which secreted BDNF continuously, providing a model for neuron-like cells. Thus, irNCs offer promise in investigating various neural diseases by using neural-like cells derived directly from the patient of interest.
\end{abstract}

Correspondence to: Professor Hengzhu Zhang, Department of Neurosurgery, Northern Jiangsu People's Hospital, 98 Nantong West Road, Yangzhou, Jiangsu 225001, P.R. China

E-mail: zhanghengzhu@sina.com

*Contributed equally

Abbreviations: irNCs, induced-resembled neuronal cells; Ascl1, achaete-scute homolog 1; Neurod1, neuronal differentiation factor 1; Myt11, myelin transcription factor 1; Brn2/POU3F2, brain protein 2; h-BDNF, human brain-derived neurotrophic factor

Key words: genetic lineage conversion, A375, induced-resembled neuronal cells, human brain-derived neurotrophic factor

\section{Introduction}

Established animal models of neuron development are well characterized, however, despite providing key insights into mechanisms, translating these findings to humans is limited by the absence of human neurons for laboratory investigations. However, a large-scale drug screening process generally requires a large supply of cells to ensure the reproducibility of the assay. From the perspective of neurosurgery, it is not routinely possible to isolate analogous populations of human neurons, or to isolate and fully examine central neurons. Therefore, a novel strategy of neuronal cell-line development in describing human biology is required. There has been increasing focus on the development of effective methods to generate neurons with a region-specific phenotype and mature functional properties. Lineage reprogramming makes it possible to generate these resembled neurons.

The potential of transcription factors in inducing somatic cells into differentiated cells has been known since the demonstration of the ability of MyoD to transform fibroblasts into muscle-like cells (1). In 2006, induced pluripotent stem cells (iPSCs) were obtained for the first time by the introduction of four genes, which were able to reprogram somatic mouse cells into pluripotent stem cells (2). One year later, it was demonstrated that human fibroblast cells can also be reprogrammed (3). This novel source of pluripotent cells has accelerated the number of investigations in reprogramming. It has been reported that transcription factors can assist in converting between differentiated cells: Pancreatic exocrine cells are derived from pancreatic endoderm, as are $\beta$-cells (4), and the basic helix-loop-helix transcription factor, neurogenin 3 , in combination with pancreatic and duodenal homeobox 1 and Mafa, can efficiently convert pancreatic exocrine cells into $\beta$-cells in vivo (5). Previously, using neurogenin 1 (Neurog1), NeuroD1 and SRY-related high-mobility-group box 2 transcription factors, cochlear non-sensory epithelial cells were able to develop as neurons, as the ectopic expression of these factors was sufficient to induce a neuronal phenotype (6). However, neuronal induction of non-sensory cells only occurs at embryonic stages, and the efficiency of induction is relatively low. Liu et al (7) induced dopaminergic neuron-like cells from fibroblasts, and the direct reprogramming of mouse and human fibroblasts has been shown in a variety of cells, including general 
neurons $(8,9)$, dopaminergic neurons $(10,11)$, and motor neurons (12).

Vierbuchen et al (8) efficiently converted mouse fibroblasts into functional induced neuronal (iN) cells with brain protein 2 (Brn2), achaete-scute homolog 1 (Ascl1) and myelin transcription factor 1 (Mytl) (8). Pang et al (9) showed that the same factors converted fetal and postnatal human fibroblasts into iN cells combined with NeuroD1. Furthermore, Ascl1 alone was sufficient to induce neurons from mouse fibroblasts, and Ascl1 was directly linked to the expansion of neural progenitors, later cell cycle exit and neural differentiation (13). The inducing of neurons by fibroblast represents the foundation of genetic lineage conversion. Son et al (12) reported that the overexpression of selected transcription factors is sufficient to convert mouse and human fibroblasts into induced motor neurons (iMNs). This involved firstly using three factors (Ascl1, Brn2 and Mytl) to obtain neuronal-like cells from fibroblasts. Following confirmation of cells with their neuronal morphology only, and without markers of motor neurons, eight candidate transcription factors, which are involved in varying stages of motor neuron specification were added. As expected, a significant number of motor cells emerged with characteristics of cultured embryonic motor neurons. These cells exhibited the expected electrophysiological properties and formed functional synaptic connections with myotubes. In addition to these findings, Son et al (12) described three limitations: Only small quantities of neurons were generated and synapse formation was limited; iMNs depend on the level of brain-derived neurotrophic factor (BDNF), thus if the level of BDNF in the medium is insufficient, the survival rate of the iMNs is negligible; and the majority of the primary cells were collected from embryos, presenting an unavoidable deficiency in terms of application.

Following examination of the previous literature (14-16), to overcome these limitations, the present study aimed to convert melanoma cells of the A375 cell line into induced-resembled neural cells (irNCs) by introducing a combination of four neuronal transcription factors, Ascl1, Neurod1, Mytl and Brn2. In addition, to promote the induction of cells, human (h)-BDNF was introduced with these factors. Whole-cell patch clamp examinations revealed that these cells exhibited neuronal membrane properties and the ability to fire action potentials. In addition, primary tumor markers were largely downregulated in vitro.

The irNCs exhibited typical properties of neurons in terms of morphology, gene expression and electrophysiology. The data indicated that Myt1 alone or the four-factor set of transcription factors were sufficient to reprogram the A375 cells into neurons. The five-factor set reprogrammed the melanoma cells into functional neuronal cells expressing BDNF. These methods may facilitate the robust generation of specific human neurons for disease modeling, drug screening, or future applications in regenerative medicine.

\section{Materials and methods}

Cell culture and treatment. The A375 human melanoma cell line was obtained from GeneChem Co., Ltd. (Shanghai, China) and maintained in Dulbecco's modified Eagle's medium (Gibco; Thermo Fisher Scientific, Inc., Waltham, MA, USA) supplemented with $10 \%$ fetal bovine serum (FBS; Gibco; Thermo Fisher Scientific, Inc.) and antibiotics (100 U/ml penicillin and $100 \mathrm{mg} / \mathrm{ml}$ streptomycin (Gibco; Thermo Fisher Scientific, Inc.). All cell types were incubated in a $5 \% \mathrm{CO}_{2}$ humidified incubator at $37^{\circ} \mathrm{C}$. At $24 \mathrm{~h}$ prior to infection, $293 \mathrm{~T}$ cells in the exponential phase of growth were re-plated into a $15 \mathrm{~cm}$ dish at a density of $1.2 \times 10^{7} / 20 \mathrm{ml}$. The A375 cells were at a density of $3 \times 10^{6}$ cells in a $10 \mathrm{~cm}$ dish.

Lentivirus production and lentivirus transduction. The lentiviruses were produced from 293 T cells (GeneChem Co., Ltd., Shanghai, China), according to a previously described protocol (17). Briefly, genes encoding the human transcription factors, Ascl1, Brn2, Myt1 or Neurod1 were cloned into the lentiviral vector, pGC-LV-GV166 (GeneChem Co., Ltd., Shanghai, China). The description of the plasmid is described at http://www.genechem.com.cn/Zaiti.aspx?zt=GV166. To generate the pGC-LV-GV166-Ascl1, -BDNF, -Myt1 and -Neurod1, the 293 T cells ( $3 \times 10^{6}$ cells) at 70-80\% confluence were triple transfected with the pGC-LV-GV166 plasmid (GeneChem, Co., Ltd; $20 \mu \mathrm{g}$ ), the pHelper 1.0 plasmid (15 $\mu \mathrm{g}$; Invitrogen; Thermo Fisher Scientific, Inc., Waltham, MA, USA) and the pHelper 2.0 plasmid $(10 \mu \mathrm{g})$ using Lipofectamine 2000 (Invitrogen; Thermo Fisher Scientific, Inc.) with a total transfection volume of $100 \mu \mathrm{l}$. DNA sequencing was performed by GeneChem to verify the sequence of the insert, and the identities were $100 \%$. The amplified sequence was digested with AgeI and BamHI (both purchased from New England Biolabs, Inc., Hitchin, UK), and the coding regions of the Ascl1, BDNF, Myt1 and Neurod1 genes were amplified using polymerase chain reaction analysis (PCR), with the primers shown in Table I. The transfection medium was replaced with regular $\alpha$-MEM/10\% FBS medium $8 \mathrm{~h}$ following transfection. After an additional $48 \mathrm{~h}$, the $293 \mathrm{~T}$ supernatant was harvested and sterile-filtered, and was centrifuged at $4,000 \mathrm{x}$ g for $10 \mathrm{~min}$ at $4^{\circ} \mathrm{C}$ using a Centricon Plus-20 Centrifugal Filter device (EMD Millipore, Billerica, MA, USA) to obtain concentrated virus, the titer of which was estimated using PCR. PCR analysis was performed to confirm the result of the lentivirus production of Ascl1, BDNF, Myt1 and Neurod1 in the transfected 293 T cells. The A375 cells $\left(3 \times 10^{6}\right.$ cells) were then transduced with either the Ascl1-, BDNF-, Myt1- or Neurod1-overexpression lentivirus or the control lentivirus for $48 \mathrm{~h}$ at a multiplicity of infection (MOI) of 10 in regular $\alpha$-MEM/10\% FBS medium to generate the irNCs.

Immunofluorescence staining. For immunofluorescence staining, the cells were washed twice with phosphate-buffered saline (PBS) and fixed with 4\% paraformaldehyde for $10 \mathrm{~min}$. The cells were then washed twice with PBS and blocked for $45 \mathrm{~min}$ at room temperature with PBS containing $1 \%$ bovine serum albumin (Gibco; Thermo Fisher Scientific, Inc., Shanghai, China) and $0.1 \%$ Triton X-100. Primary antibodies were used at dilutions recommended by the manufacturer. rabbit anti-microtubule-associated protein 2 (MAP2; Santa Cruz Biotechnology, Inc., Dallas, TX, USA;1:1,000). The following anti-human antibodies used were: Mouse anti-tubulin $\beta 3$ (Tuj1; cat. no. MMS-435P-250, Covance, Inc., Princeton, NJ, USA; 1:800), mouse anti-neuronal nuclear (NEUN; cat. no. MAB377, EMD Millipore; 1:200) and rabbit anti-microtubule-associated 
Table I. Primers used for polymerase chain reaction amplification of the coding region of gene.

\begin{tabular}{ll}
\hline Gene ID & \multicolumn{1}{c}{ Primers (5'-3') } \\
\hline MYT1L (3247-2)-F & AGGTCGACTCTAGAGGATCCCGCCACCATGGAGGTGGACACCGAGGAGAAGCGGCAT \\
MYT1L (3247-2)-R & AGTCCATGGTGGCGACCGGGACCTGAATTCCTCTCACAGCCTGCTTTA \\
NEUROD1 (3268-7)-F & AGGTCGACTCTAGAGGATCCCGCCACCATGACCAAATCGTACAGCGA \\
NEUROD1 (3268-7)-R & AGTCCATGGTGGCGACCGGATCATGAAATATGGCATTGAGC \\
ASCL1 (3269-1)-F & AGGTCGACTCTAGAGGATCCCGCCACCATGGAAAGCTCTGCCAAGATGG \\
ASCL1 (3269-1)-R & AGTCCATGGTGGCGACCGGGAACCAGTTGGTGAAGTCGAG \\
POU3F2 (3270-6)-F & AGGTCGACTCTAGAGGATCCCGCCACCATGGCGACCGCAGCGTCTAAC \\
POU3F2 (3270-6)-R & AGTCCATGGTGGCGACCGGCTGGACGGGCGTCTGCAC \\
BDNF (4916-2)-F & AGGTCGACTCTAGAGGATCCCGCCACCATGACCATCCTTTTCCTTAC \\
BDNF (4916-2)-R & AGTCCATGGTGGCGACCGGTCTTCCCCTTTTAATGGTC
\end{tabular}

MYT1L, myelin transcription factor 1; NEUROD1, neuronal differentiation factor 1; ASCL1, achaete-scute homolog 1; POU3F2; brain protein 2; BDNF, brain-derived neurotrophic factor; $\mathrm{F}$, forward; $\mathrm{R}$, reverse.

Table II. Primers used for quantitative polymerase chain reaction analysis of exogenous and endogenous genes.

\begin{tabular}{|c|c|}
\hline Gene ID & Primers $\left(5^{\prime}-3^{\prime}\right)$ \\
\hline BDNF (NM_170735)-F & GTGTGACAGTATTAGTGAGTGG \\
\hline BDNF (NM_170735)-R & CTTGGTCTCGTAGAAGTATTGC \\
\hline SYN1 (NM_006950)-F & TGCTCAGCAGTACAACGTACC \\
\hline SYN1 (NM_006950)-R & GACACTTGCGATGTCCTGGAA \\
\hline RBFOX3 (NM_001082575)-F & CCAAGCGGCTACACGTCTC \\
\hline RBFOX3 (NM_001082575)-R & CGTCCCATTCAGCTTCTCCC \\
\hline TUBB3 (NM_006086)-F & GAACCCCAGGCAGCTAGA \\
\hline TUBB3 (NM_006086)-R & TCCCAGAACTGTGGACGC \\
\hline MAP2 (NM_001039538)-F & CGTAACCCTGTTTTAGTGC \\
\hline MAP2 (NM_001039538)-R & ACCAACAATCTGTAAACCCT \\
\hline MIA (NM_006533)-F & GTCAGGGGTGGTCCTATGC \\
\hline MIA(NM_006533)-R & GGTCAGGAATCGGCAGTCG \\
\hline IGF2BP3 (NM_006547)-F & ATCCGTATCCAAGCAGAAACC \\
\hline IGF2BP3 (NM_006547)-R & GACTTACAAGCCGCAGAGG \\
\hline CHI3L1 (NM_001276)-F & AAGCAACGATCACATCGACAC \\
\hline CHI3L1 (NM_001276)-R & TCAGGTTGGGGTTCCTGTTCT \\
\hline S100B (NM_006272)-F & TGGCCCTCATCGACGTTTTC \\
\hline S100B (NM_006272)-R & ATGTTCAAAGAACTCGTGGCA \\
\hline GAPDH-F & TGACTTCAACAGCGACACCCA \\
\hline GAPDH-R & CACCCTGTTGCTGTAGCCAAA \\
\hline
\end{tabular}

BDNF, brain-derived neurotrophic factor; SYN1, synapsin 1; RBFOX3, RNA binding protein, Fox-1 homolog 3; TUBB3, tubulin $\beta 3$; MAP2, microtubule-associated protein 2 ; MIA, melanoma inhibitory activity; IGF2BP3, insulin-like growth factor 2 mRNA binding protein 3; CHI3L1, chitinase 3-like 1; GAPDH, glyceraldehyde 3-phosphate dehydrogenase; F, forward; R, reverse.

protein 2 (MAP2; cat. no. sc-25663, Santa Cruz Biotechnology, Inc., Dallas, TX, USA; 1:1,000). Secondary antibodies labeled with either Alexa488 or Alexa555 were obtained from Invitrogen; Thermo Fisher Scientific, Inc.

Reverse transcription-quantitative $(R T-q) P C R$. Cells were collected by centrifugation at $2,000 \mathrm{rpm}$ for $5 \mathrm{~min}$. Total RNA was extracted using an RNAeasy Mini kit (Qiagen,
Hilden, Germany), according to the manufacturer's protocol. Reverse transcription of $2 \mu \mathrm{g}$ RNA to cDNA was performed using Moloney-murine leukemia virus reverse transcriptase (Promega, Corp., Madison, WI, USA) and Oligo dT. A full description of the primer sequences are listed in Table II. RT-qPCR was performed using $1 \mu \mathrm{l}$ of cDNA, $8 \mu \mathrm{l}$ of RNase-free $\mathrm{H}_{2} \mathrm{O}, 10 \mu \mathrm{l}$ SYBR premix ex taq (Takara Bio, Inc., Otsu, Japan), $1 \mu \mathrm{l}$ of each primer and deionized distilled 
water, in a $20 \mu 1$ total reaction volume. The PCR program used was as follows: Step 1: $95^{\circ} \mathrm{C}$ for $30 \mathrm{sec}$; Step 2: $95^{\circ} \mathrm{C}$ for $5 \mathrm{sec}$; Step 3: $60^{\circ} \mathrm{C}$ for $30 \mathrm{sec}$; Step 4: $95^{\circ} \mathrm{C}$ for $15 \mathrm{sec}$; Step 5: $55^{\circ} \mathrm{C}$ for $30 \mathrm{sec}$; and Step 6: $95^{\circ} \mathrm{C}$ for $15 \mathrm{sec}$. Steps 2-3 were repeated 45 times. A Nanodrop 2000/2000C spectrophotometer (Thermo Fisher Scientific, Inc.) was used for the quantitative analysis, and S-100B (MIA, YKL-40) mRNA levels were semi-quantitatively assessed as the S100B (MIA, YKL-40)/glyceraldehyde-3-phosphate dehydrogenase mRNA ratio.

Assessment of electrophysiological function. The whole-cell configuration of the patch-clamp technique was performed at room temperature using an Axopatch $700 \mathrm{~A}$ amplifier (Molecular Devices LLC, Sunnyvale, CA, USA) with PCLAMP 9 software (Molecular Devices LLC). The pipette internal solution contained $140 \mathrm{mM} \mathrm{KCl}, 10 \mathrm{mM}$ 4-(2-hydroxyethyl)piperazine-1-ethanesulfonic acid (HEPES), $1 \mathrm{mM} \mathrm{MgCl}, 10 \mathrm{mM}$ ethylene glycol tetra-acetic acid, $1 \mathrm{mM} \mathrm{NaCl}, 5 \mathrm{mM}$ phosphocreatine-Tris and $0.3 \mathrm{mM}$ $\mathrm{Na}_{2}$ ATP (pH 7.2). The bath solution contained $140 \mathrm{mM} \mathrm{NaCl}$, $10 \mathrm{mM}$ glucose, $10 \mathrm{mM}$ HEPES, $1 \mathrm{mM} \mathrm{MgCl} \mathrm{m}_{2}$ and $1 \mathrm{mM}$ $\mathrm{CaCl}_{2}$ ( $\mathrm{pH} 7.35$ ). For the current-clamp experiments, the cells were held at $-82 \mathrm{mV}$, and step currents were injected to elicit an action potential. For the voltage-clamp experiments, the cells were held at $-50 \mathrm{mV}$, and a series of depolarizing $20 \mathrm{mV}$ voltage steps between -70 and $+30 \mathrm{mV}$ was applied every $2 \mathrm{sec}$. For the sodium channel blocking experiment, $200 \mathrm{nM}$ tetrodotoxin (TTX) was used. Analyses were performed using Origin (OriginLab Corporation, Northampton, MA, USA) and PCLAMP 9 software (Molecular Devices LLC).

Statistical analysis. All data were expressed as the mean \pm standard deviation. The Student's t-test was used for comparisons between two groups (Graphpad Software, San Diego, CA, USA). $\mathrm{P}<0.05$ was considered to indicate a statistically significant value; $\mathrm{P}<0.01$ was considered to indicate a highly statistically significant value.

\section{Results}

Human melanoma cells are converted to irNCs by the introduction of neuronal transcription factors. The widely reported A375 human melanoma cells were used as source cells in the present study. To determine whether human cancer cells can be reprogrammed into irNCs, the A375 cells were infected with lentiviral vectors expressing four reprogramming factors: Ascl1, Neurod1, Mytl and Brn2. In addition, in order to obtain mature neurons, the h-BDNF gene was cloned and packaged into the lentivirus particles. Following transduction of the empty virus into the melanoma cells, it was found that the infection efficiency of the melanoma cells was $\sim 90-100 \%$ when the MOI reached 10 (Fig. 1A and B). No significant changes were observed in the infection efficiency by increasing the viral titer. Following transduction with the lentiviral vector $(\mathrm{MOI}=10)$ for 5 days, enhanced green fluorescence protein (EGFP) fluorescence intensity remained high, and an increasing trend was observed in the subsequent 4 weeks. Of note, the experiments in the present study demonstrated that $\mathrm{h}$-BDNF was not required for the reprogramming progress.
Using the five-transcription-factor protocol, it was possible to reprogram the A375 cells into irNCs, which stained positive for the general neuron-specific marker, Tuj1, consistently expressed h-BDNF and generated action potentials.

As the Myt1 gene fragment was larger (3,598 bp), compared with the other transcription factors, the present study confirmed that the number of remaining cells was sufficient for experiments downstream following puromycin screening. After 7 days of screening, $\sim 60-70 \%$ of the cells remained and the majority of cells began to exhibit features of axons (Fig. 1C and D).

Essential requirements of Ascll, Neurod1, Brn2 and h-BDNF for the direct reprogramming of A375 cells into irNCs. Following Myt1 transduction, the present study examined whether the remaining cells were also able to be induced into neuronal-like cells with the other transcription factors, Ascl1, Neurod1 and Brn2. On day 7, between three and five neuronal-like colonies appeared in the donor cells per 500 cells following vaccination. These colonies had marked proliferating capability and exhibited typical bipolar neuronal morphology, but axonal synapse generation was rare (Fig. 2A-C). Based on the above results, a five-factor group was established, with h-BDNF added to the original four-factor group to enhance cellular differentiation in vitro. The number of neural-like colonies increased significantly for 4 weeks. Neuronal morphology in the five-factor group was mature and present for a longer duration, compared with the others (Fig. 2D-F). Following infection with the EGFP virus alone, no neuronal cells were generated during the same time-frame, and almost all the A375 cells died due to the puromycin treatment. Mytl alone was able to induce neuronal morphology, and the relative contribution each of the three factors(Ascl1, Brn2, Neurod1) were determined. The addition of Ascl1, Brn2, Neurod1 or two in combination did not increase the efficiency of neuronal differentiation (Fig. 3), however, morphologies increased in complexity. The cells transduced with all four factors together exhibited the most mature neuronal morphologies.

At the beginning of the first 3 days, the four- and five-factor groups were Tuj1-positive. The level of Tuj1 in the five-factor group was significantly higher, compared with that in the four-factor group. On days 7 and 14, the cellular morphology of the five-factor group was more mature and complete, compared with that of the four-factor group; the four-factor cells exhibited a simple bipolar morphology, whereas the five-factor cells appeared tripolar or with multi-axonal differentiation (Fig. 4A-C).

Characterization of irNCs generated from melanoma cells. To understand the induction process in detail, the present study investigated whether irNCs expressed neuronal-specific markers at the mRNA and protein levels between days 3 and 30. Anti-NEUN antibody is specifically bound with nerve cell nucleus antigen, indicating the positive area of the nucleus. It is expressed in normal brain tissues, including pyramidal neurons and granule neurons, with the exception of cerebellar Purkinje cells. Of note, NEUN is only expressed in mature neurons. Compared with NEUN, MAP2 is positively expressed in normal brain tissue and neurons in the cytoplasm. However, a previous study showed that MAP2 
A

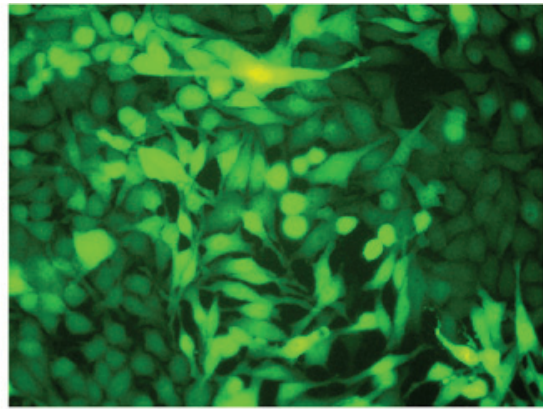

C

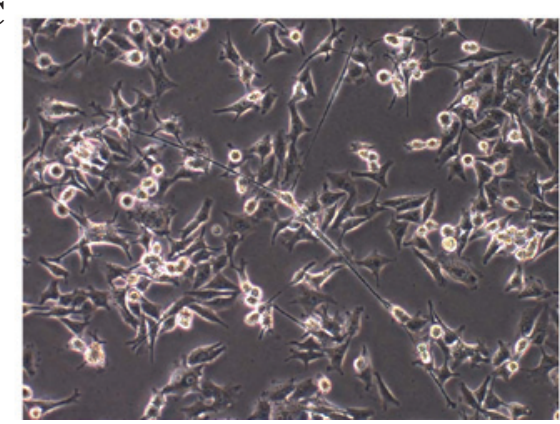

B

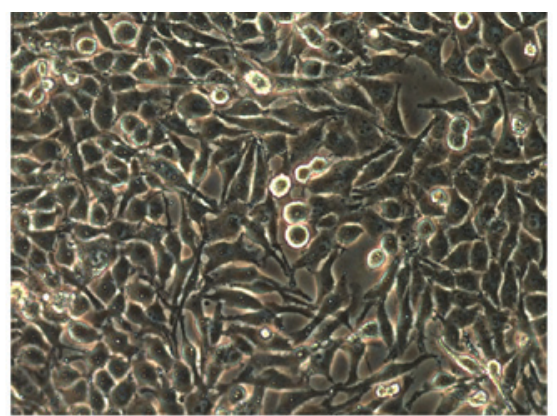

D

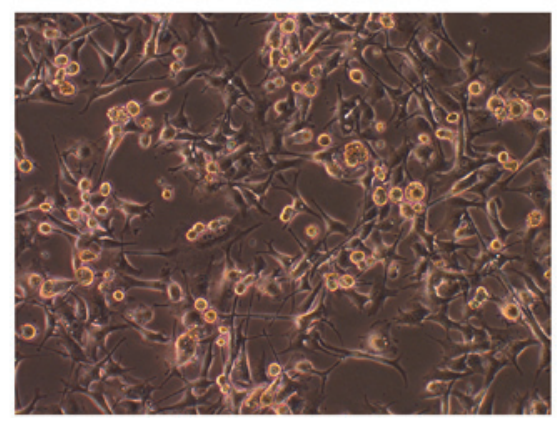

Figure 1. Rapid generation of induced-resembled neuronal cells from A375 cells. (A and B) A375 melanoma cells infected by the empty viral vector with enhanced green fluorescent protein. The infection rate reached $>95 \%$ with an $\mathrm{MOI}=20$. The designated concentrations were derived as follows: $\mathrm{MOI}=1$, $1 \times 10^{8} \mathrm{TU} / \mathrm{ml}$ of lentivirus $+0.1 \mu \mathrm{l}$ lentivirus solution; $\mathrm{MOI}=10,1 \times 10^{8} \mathrm{TU} / \mathrm{ml}$ of lentivirus $+1 \mu 1$ lentivirus solution; $\mathrm{MOI}=100,1 \times 10^{8} \mathrm{TU} / \mathrm{ml}$ of lentivirus $+1 \mu 1$ lentivirus solution). The A375 cells were readily infected by the lentivirus. (A) Green fluorescence imaging was used to identify individual cells. (B) The same field was in white. (C and D) Following screening using puromycin, the percentage of cells infected by myelin transcription factor 1, which remained of the original group was $\sim 65 \%$, and the majority of cells exhibited axon-like changes. Magnification, x200. MOI, multiplicity of infection. (C) The cells prior to screening. (D) The cells following screening.
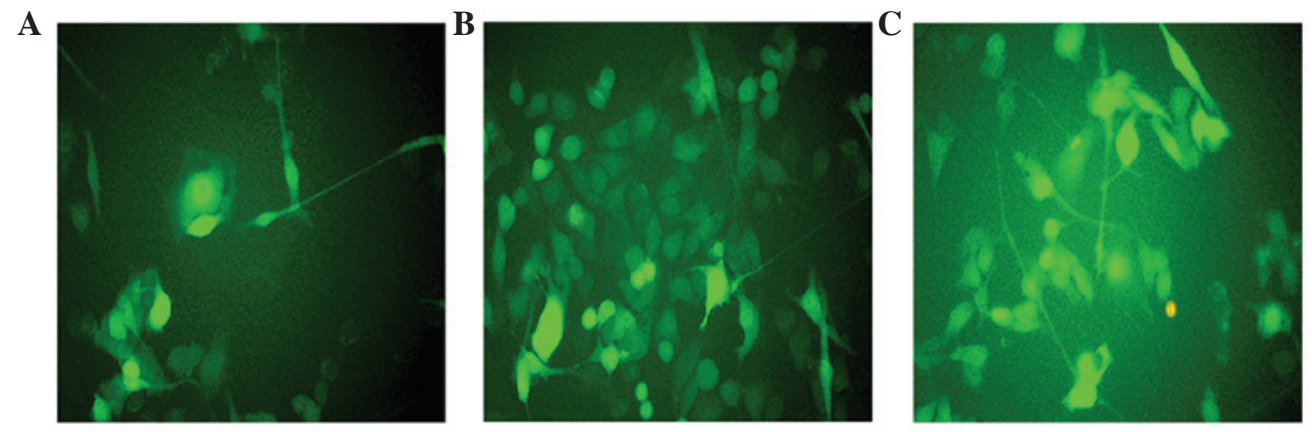

D
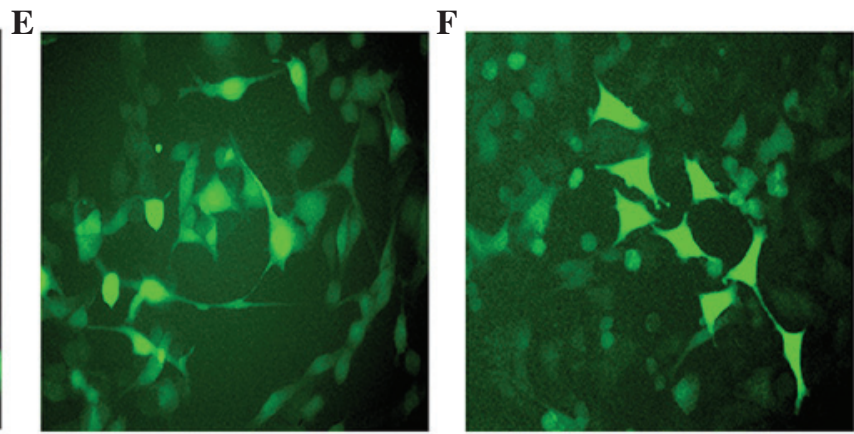

Figure 2. Green fluorescence imaging following infection with ABMN factors. Green fluorescence imaging (A) 7, (B) 14 and (C) 21 days following infection with ABMN factors. Fraction of cells stained by green fluorescence (enhanced green fluorescence protein positive cells) (D) 7, (E) 14 and (F) 21 days following five factor (ABMN+ human brain-derived neurotrophic factor) gene transduction. All images were captured under a microscope (magnification, x200).

is also expressed in low-grade neural epithelial tumors, particularly oligodendrocytes. Therefore it is suggested that MAP2 is not only neuronal marker, but also acts as a marker of glial precursors. Therefore, the present study assessed immunoreactivity with the Tuj1 antibody. The Tuj1 antibody has been widely confirmed to identify neurons without the identification of glial cells. Therefore, Tuj1 antibody staining is widely used for the immunization of nerve cells and as a 
Tuj1 (+) cells

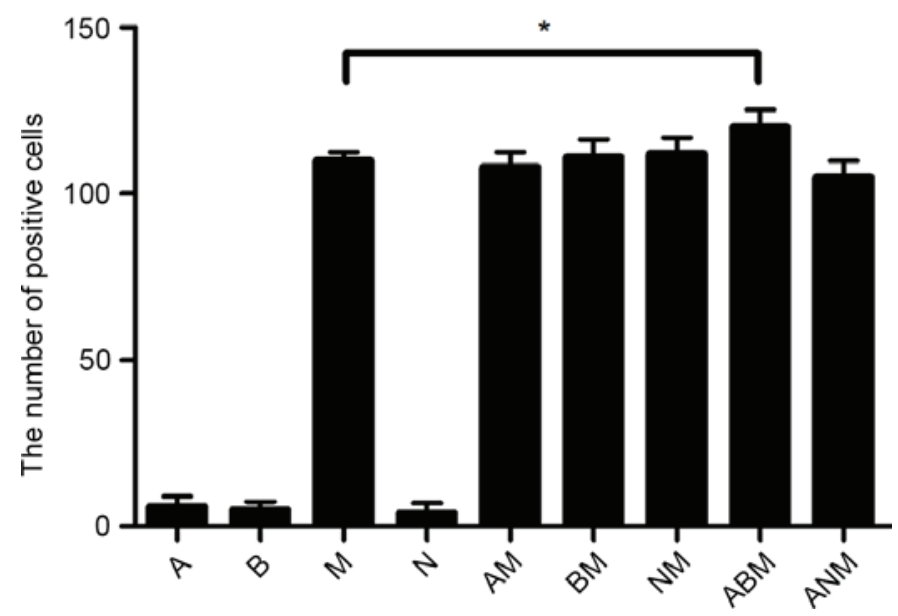

Figure 3. Importance of transduced factors in the reprogramming procedure. Quantification of Tuj1-positive cells with the indicated factor combinations of the brain protein 2 (B), achaete-scute homolog 1 (A), myelin transcription factor 1 (M) and neuronal differentiation factor 1 (N) genes 7 days following infection. Data are presented as the mean \pm standard deviation of three randomly selected images (AxioCam), captured with a 200x objective on a Zeiss Axio Observer microscope. The total quantity of virus was constant between the different factor combinations, and the same numbers of A375 cells were seeded in each condition prior to infection. ${ }^{*} \mathrm{P}<0.05$. Tuj1, tubulin $\beta 3$.
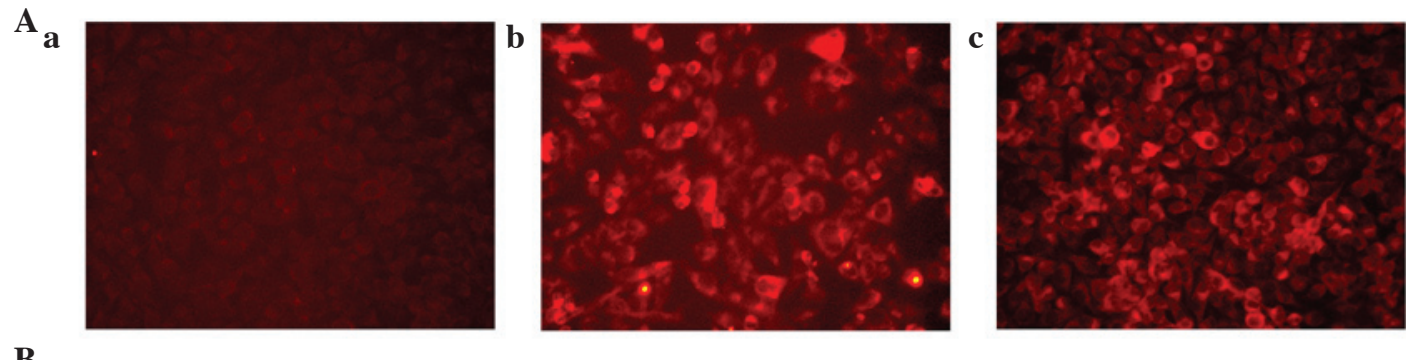

B
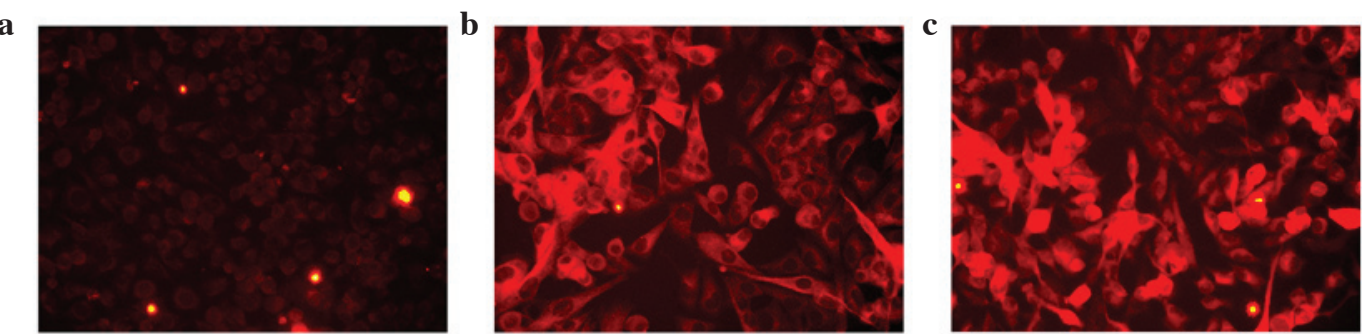

C
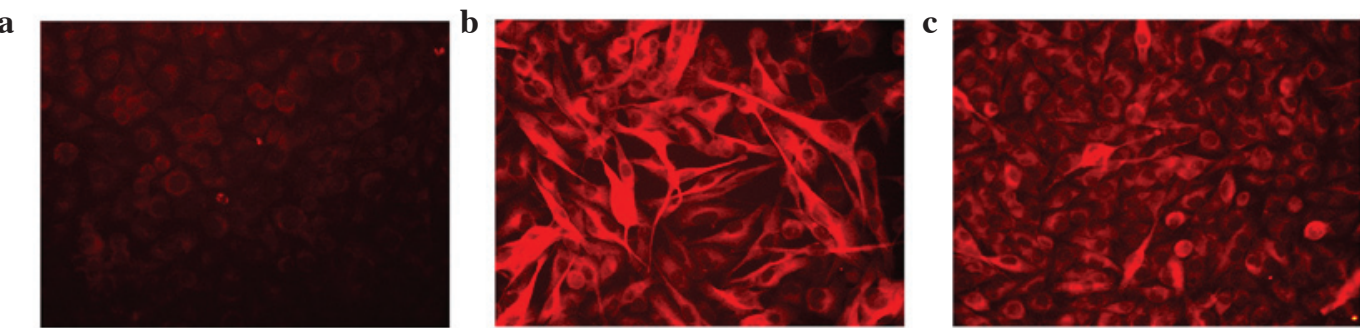

Figure 4. h-BDNF improves complex morphologies of irNCs. (A) At 1 week post-empty virus transduction, the cells in the five-factor and four-factor-infected cells were stained with Tuj1. (B) At 2 weeks post-empty virus transduction, the ABMN + h-BDNF and ABMN irNCs exhibited neuronal morphologies and expressed Tuj1. (C) Immunofluorescence images of the control, five-factor and four-factor groups on day 21. The (a) panels demonstrate the empty-virus group; the (b) panels demonstrate the five-factor group; and the (c) panels demonstrate the four-factor group. All images were captured under a microscope (magnification, x200). irNCs, induced resembled neuronal cells; h-BDNF, human brain-derived neurotrophic factor; Tuj1, tubulin $\beta 3$.

reference detector of neurons. In the present study, several neuron-specific markers showed high mRNA expression levels. The maximum expression occurred on day 14 during the cellular differentiation. The changes in the expression levels among the three groups were maintained for almost 4 weeks. By contrast, following transduction with the empty virus, no specific markers were observed during the same time frame. The difference between the three groups 
A

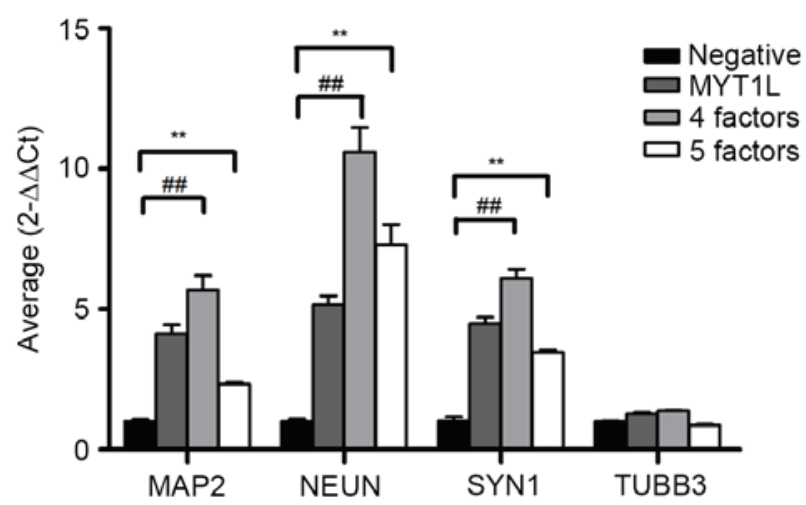

C

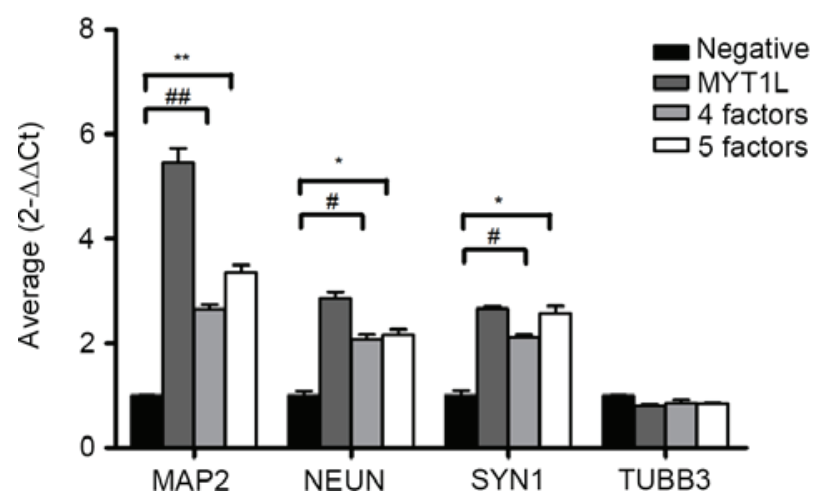

B 2 weeks after infection

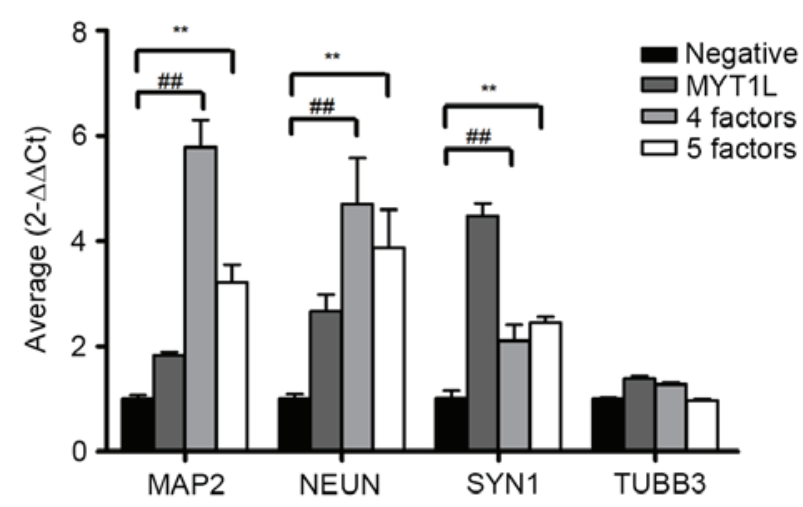

D

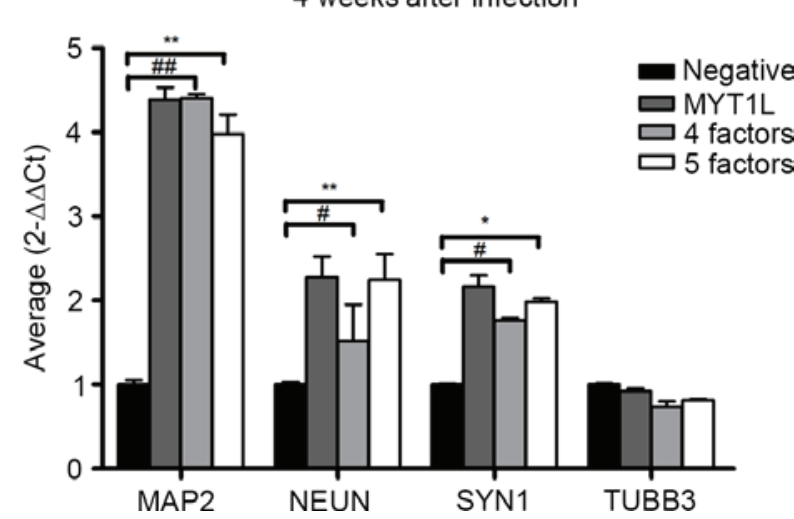

Figure 5. Results of reverse transcription-quantitative polymerase chain reaction analysis of A375 irNCs. Statistical analyses of the expression levels of neuron-specific markers in the empty-virus, four-factor and five-factor groups at week (A) 1, (B) 2, (C) 3 and (D) 4 . The levels of MAP2, NEUN and SYN1 from the four-factor and five-factor group were higher, compared with the negative control in the 4 weeks. Data are expressed as the mean \pm standard deviation. ${ }_{\# \# * *} \mathrm{P}<0.01$ and ${ }^{\# *} \mathrm{P}<0.05$. irNCs, induced resembled neuronal cells; MAP2, microtubule-associated protein 2; NEUN, neuronal nuclei; SYN1, synapsin 1.

remained for almost four weeks (Fig. 5). Notably, the irNCs overexpressed h-BDNF at a high level during the 4 weeks, with an increasing trend in vitro (Fig. 6).

In order to ensure that the irNCs acquired neuronal properties, Tuj1-positive cells were used as a control. At 2 weeks post-induction, the irNCs in the single factor group, which expressed Tuj1, did not detect the exhibit cellular action potential firing. The irNCs in the four-factor group exhibited a single spike. The irNCs in the five-factor group fired repetitive action potentials with a regular spiking pattern, which was suppressed by the TTX-dependent sodium channel-specific inward current. However the action potential amplitude and frequency of the irNCs remained different, compared with those of the embryonic stem cell-derived neurons (Fig. 7A-D).

The ABMN (ㅅscl1, $\underline{B} r n 2, \underline{M y t 1 l,}$ Neurod1) transcription factor and h-BDNF reduce the degree of malignancy of melanoma cells and increase migration in vitro. To determine whether ABNM and h-BDNF decreased the tumorigenicity of the melanoma cells, the present study examined S-100B, melanoma inhibiting protein (MIA) and YKL-40 in vitro. The results revealed that the S-100B and MIA expressed in the four-factor and five-factor groups were markedly reduced for almost 4 weeks, suggesting a decrease in tumor malignancy. In addition, the levels of S-100B and MIA on day 14 were significantly lower, compared with those in the empty virus group
$(\mathrm{P}<0.05)$. Of note, the overexpression of YKL-40 indicated a significant enhancement of cell migration and differentiation (Fig. 6).

\section{Discussion}

Using a protocol previously developed by Son et al (12) for the induction of motor neuron cells from mouse and human fibroblast cells, the present study aimed to reprogram A375 cells. To overcome the three limitations reported by Son et al (12), adult human epidermal keratinocytes were selected and MOI measurements were performed prior to transfection. However, with $>80 \%$ of cells infected, the MOI had reached 100 , and the majority of cells were unable to survive transduction with a high titer virus, leading to rapid apoptosis. Pressure to improve the feasibility of the experimental procedure required a novel type of cell with superior vitality and the ability to withstand lentivirus transduction. In accordance with associated literature and clinical requirements, the cells used for lineage conversion require four basic conditions to be met: The cell can be harvested readily and show enhanced vitality; the cell can withstand repeated transfection with multiple factors and the number of screened cells is sufficient for downstream experiments; following reprogramming, more single target pedigree can be obtained; experimentally transfected cells can secrete high levels of nerve growth factor 
A

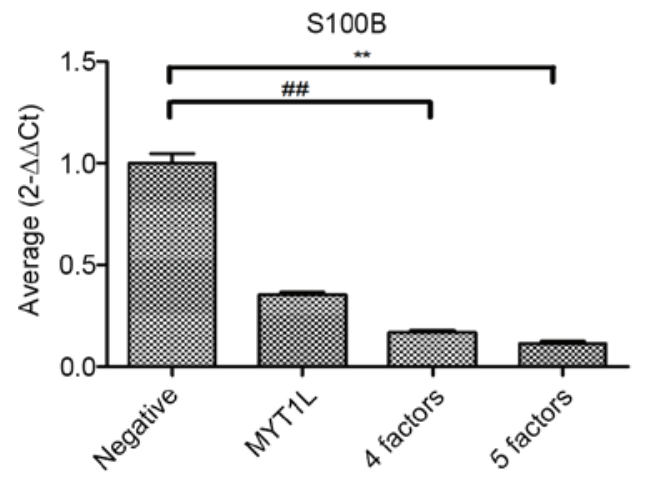

C

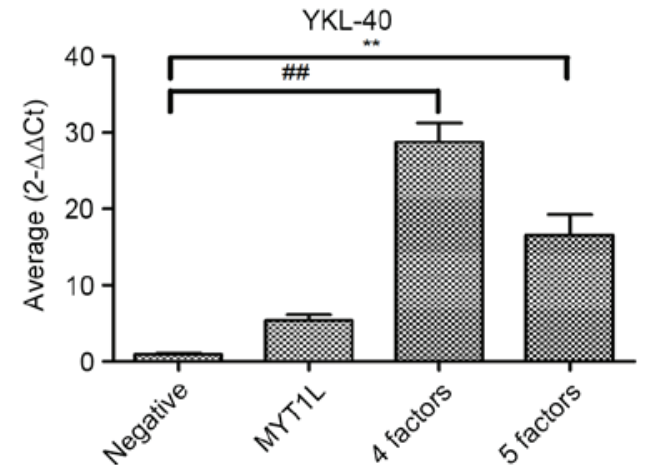

B

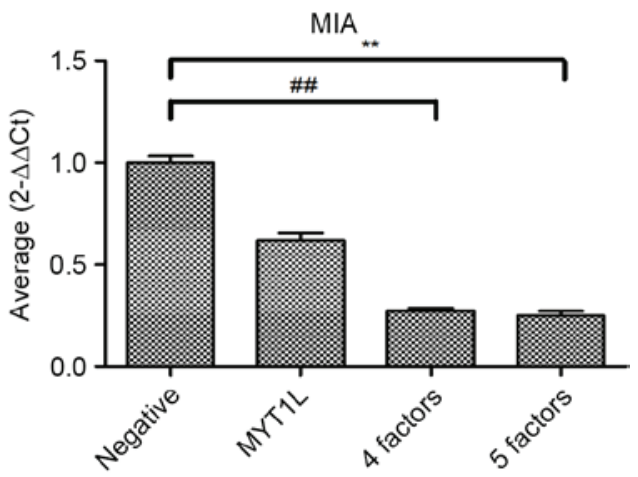

D

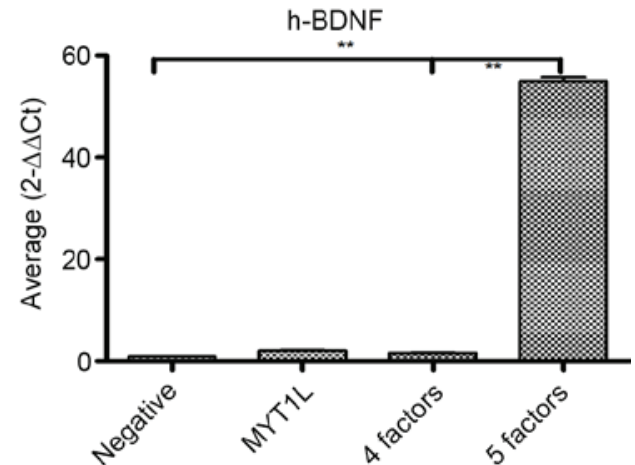

Figure 6. Markers of malignant tumor. (A) S-100B and (B) MIA, were examined by RT-qPCR analysis on day 14. Reprogramming genes reduced the malignancy of the irNCs. (C) Analysis of YKL-40 by RT-qPCR analysis on day 14, the reprogrammed cells induced migration. (D) Analysis of h-BDNF by RT-qPCR analysis on day 4 showed the infected gene was expressed consistently. All data are expressed as the mean \pm standard deviation. ${ }^{* *} \mathrm{P}<0.01,{ }^{\# \#} \mathrm{P}<0.01$. RT-qPCR, reverse transcription-quantitative polymerase chain reaction; MIA, melanoma inhibiting protein; YKL-40, chitinase 3-like 1; h-BDNF, human brain-derived neurotrophic factor.

A

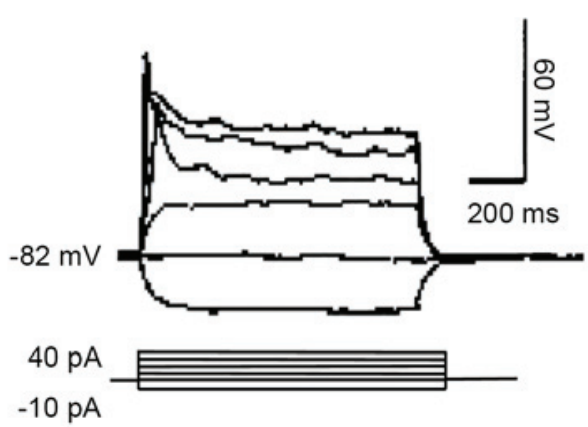

B

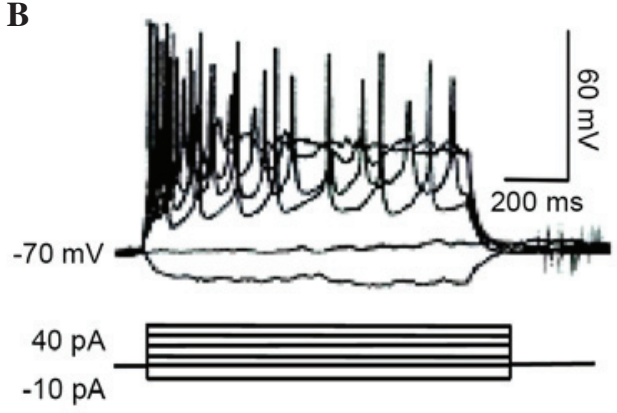

C

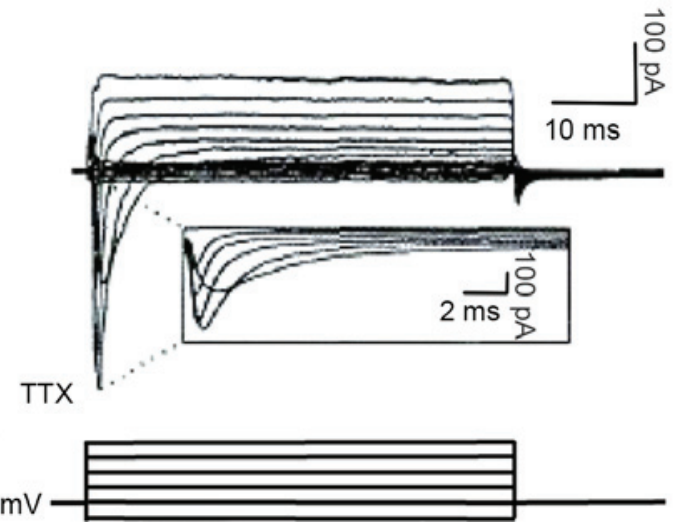

D

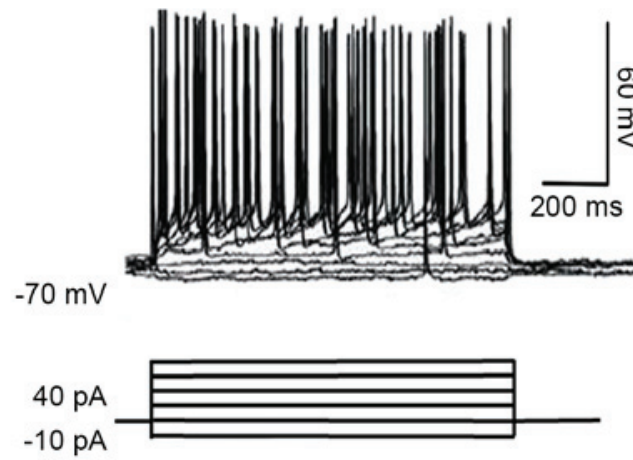

Figure 7. Membrane properties of irNCs. (A) Membrane properties of four-factor irNCs. Single, action potential-like responses were recorded following current injections between -10 and $40 \mathrm{pA}$, in $10 \mathrm{pA}$ steps. (B) Typical action potentials recorded in the five-factor group. The five-factor group of mature irNCs under the depolarization current released action potentials. (C) In the voltage-clamp recording, an inward current was induced by voltage stimulation, with the membrane potential held at $-50 \mathrm{mV}$ and voltage steps between -70 and $30 \mathrm{mV}$, which was inhibited by TTX. (D) A gap remained between the action potential amplitude and frequency between the five-factor group and embryonic stem cell-derived neurons. irNCs, induced resembled neuronal cells; TTX, tetrodotoxin. 
for a long period to reduce dependence on exogenous addition in medium and create favorable conditions for use in vitro.

The microenvironment is an important source of signals, which program mature cells to adopt a particular fate and migratory path, however, its potential to reprogram and restrict multipotent tumor cell fate and invasion remains to be fully elucidated. h-BDNF is essential for promoting the survival of neurons in the central nervous system (18). A variety of experiments in vivo have confirmed that BDNF can protect neurons from various injuries. Pencea et al (19) showed that, in glutamate-induced neuronal apoptosis confirmed in vitro, BDNF acts against excitatory amino acid neurotoxicity, predominantly via the phosphoinositide 3-kinase (PI3K)/Akt and Ras/mitogen-activated protein kinase pathways. Xia et al (20) also confirmed that BDNF protects neurons from apoptosis, and its protective effect is mediated through the extracellular signal-regulated kinase (ERK) and PI3K pathway (20). Neurotrophins participate in regulating the survival, differentiation and target innervation of many neurons. Mullen et al (21) discovered that the inhibition of Ras, p38, PI3K or Akt signaling reduced or eliminated the BDNF-mediated increase in neurite outgrowth, whereas inhibition of mitogen-activated protein kinase kinase (MEK)/ERK had no influence. However, the effect of PI3K inhibitors inhibited the survival of neurons in BDNF mediated, nor did ERK inhibitors. Neurite growth is considered an important event in neuronal development, synapse formation and neural regeneration, and the pharmaceutical control of neurite growth is of interest in terms of its clinical application (22). Huttner et al (23) demonstrated that BDNF promotes the neurite outgrowth of NSCs. Based on these observations, the present study hypothesized that transformation of the BDNF gene into A375 cells also promotes irNC differentiation with respect to synaptic formation and functional properties. To further confirm the neural network formation, the present study examined the expression of synapsin SYN in irNCs during differentiation. The data from the RT-PCR analysis revealed that the irNCs expressed SYN1 at $\sim 4$ weeks (Fig. 5A-D). Thus, the PI3K/Akt pathway had a decisive role in promoting neuronal survival. PI3K/Akt is also the most common activation pathway in human cancer cells (24). Following consideration of the first and third basic conditions (i.e. that the cell can be harvested readily and show enhanced vitality, and that, following reprogramming, more single target pedigree can be obtained), melanoma of skin-derived tumor cells were selected for experiments in the present study. PI3K/Akt activation can be observed in the majority of melanoma cell lines and in tissue samples at different stages of proliferation (25). It has been reported that the PI3K/Akt signaling pathway is essential in reducing apoptosis and promoting the survival of melanoma cells. $\mathrm{PI} 3 \mathrm{~K} / \mathrm{Akt}$ is closely associated with the migration, adhesion, angiogenesis and degradation of the extracellular matrix in melanoma (26). The most important melanoma, in terms of tumor origin, originates in neural crest tissue and is similar to nerve tissue.

Therefore, the present study investigated whether melanoma cells can be induced to neuron-like cells to achieve the following three objectives through lineage reprogramming technology: i) reprogramming melanoma cells into irNCs by infection of ABMN factors; ii) simultaneously promoting induced cell maturity in the neuronal lineage using h-BDNF; iii) in terms of overlapping portions of the PI3K/Akt signaling pathway between h-BDNF and melanoma, constructing a novel type of human irNC from human melanoma, which secretes BDNF continuously as a potential model to produce neurons and avoid the current limitations in stem cell modification. The four-factor group was used, and the h-BDNF gene was introduced into the induced cells. Through RT-qPCR analysis, h-BDNF in the five-factor group was markedly expressed for 4 weeks, and was almost 55 times higher, compared with the other three groups on day 14. Using this technique, a higher number of mature irNCs were obtained, compared with the other groups, and the neuronal phenotype was maintained for an extended period of time.

During the reprogramming procedure, two differences have been observed between the melanoma cells and former fibroblasts. First, in the reprogramming process of the human fibroblast-derived lineage, Wapinski et al (27) reported no significant difference between single Ascl1 and ABM groups in the expression levels of NEUN, MAP2 and Tuj1 (28). Ascl1 alone was sufficient to induce neurons from mouse fibroblasts, suggesting Ascl1 is a reprogramming factor, which promotes neuronal differentiation without proliferation (8) and is essential in the process by which neural precursor cells give rise to neuronal cell lines $(28,29)$. However, Ascl1 alone was not sufficient to induce fibroblasts into mature neurons. Neither Brn2 or Mytl alone were able to complete the inducing process, but were involved in the subsequent maturation of neurons, including the formation of action potentials and subtype differentiation. In the reprogramming of human melanoma lineages, the present study set up a specific Myt1 group for cellular screening. The cells of the Mytl group screened by puromycin had a significant tendency towards axons. Of note, the levels of neural specific markers of the Myt1 group, including MAP2, NEUN and SYN1 were detected using RT-qPCR analysis. Their levels were significantly increased, compared with those in the empty virus group, and exceeded those in the four- and five-factor groups. Therefore, the present study hypothesized that Mytl may have the same role as a target-specific factor, as with Asc1l in the fibroblast lineage. This requires further validation in vitro and in vivo. At 13-14 days post-infection with ABMN factors, cells with immature neuronal morphologies were detected. These cells expressed Tuj1, but remained functionally immature in terms of generating action potentials (Fig. 7A). Tuj1, one of the tubulin- $\beta$ family members specifically expressed in neurons, was considered to be important in maintaining the normal state of axons. The results of the RT-qPCR analysis demonstrated that, unlike the pedigree of fetal fibroblasts, Tuj1 was not expressed at a high level during the period of reprogramming melanoma. This may explain why irNCs maintained neuronal morphology only for 30 days and then degenerated day by day.

It has been increasingly reported that the tumorigenicity of direct lineage reprogramming presents a challenge. However, oncogenes may be used for reprogramming. For example, the reduced expression of tumor suppressor p53 has been found to result in more efficient cellular reprogramming (30). The majority of tumors are associated with a defective p53 
suppressor pathway, suggesting enhanced rather than reduced reprogramming efficiency of iPSCs. Certain cancer-specific genetic lesions have been shown to affect programming, although whether and how these defects affect these challenges remain to be fully elucidated. S-100B is the most widely used biomarker for malignant melanoma (31). Elevated levels of S-100B in serum suggests a poor prognosis, and S-100B has a predictive value for diagnosis of the clinical stages of malignant melanoma (32). MIA inhibits melanoma cell adhesion to fibronectin and laminin, and regulates the location of melanoma cells from the extracellular matrix (33). In the effects of metastatic tumor cells, YKL-40 is a novel inflammatory serum protein, which has been shown to be associated with the presence and prognosis of several malignancies, and acts as a regulator of cell proliferation and growth factor differentiation (34). The results of the RT-PCR analysis in the present study revealed that the irNCs from the four-factor and five-factor groups reduced the expression levels of S100B and MIA. The high level of YKL-40 may be consistent with migration, adhesion, angiogenesis and degradation through the activation of PI3K/Akt.

Compared with previous successful cases, the present study established a novel approach for formatting human neurons and improved traditional tumors, providing a foundation for overcoming the limitations identified in the analyses of human diseases, examination of human-specific genes and drug screening. Further investigations are required to understand the cellular and molecular mechanisms critical to the survival and programming of cell fate and invasion. Further investigations are also required to assess the feasibility of the induced neurons to form synaptic contacts with neural cells in the periphery and centrally. The identification of methods to detect and control tumorigenic quality, and conserve vitality and inducible capability, may assist in providing a novel type of human irNC from human melanoma cells, which secrete BDNF continuously as a potential cellular model.

In the present study, the reprogramming of A375 melanoma cells into irNCs was considered successful by characteristic neuron-like morphology, marker expression and the functional property of firing repetitive action potentials. In previous years, a number of associated experiments have demonstrated the promising potential of constructing novel culture systems in vitro by selecting candidate genes and unbiased genomes determining cellular fate (35-37). In the next decade, a variety of induced pluripotent stem cells and reprogrammed cells of human origin are likely to be reported. How to obtain the highest level of efficacy against different types of diseases as early as possible, remains to be elucidated. Lineage reprogramming technology can be used to create more homogeneous cell subtypes. Lineage reprogrammed cells, based on samples, have been used to investigate human neuropsychiatric diseases, blood diseases and tumors. Further investigations are required to advance knowledge and methods for the prevention and treatment of disease. The selection of appropriate transcription factors enables the induction of an excitatory neuronal subtype with regional specificity, however, further investigations are required to examine novel and efficient factors to reach the level of application. The model presented in the present study offers scope for future investigations of tissue engineering, disease mechanisms and drug development.

\section{Acknowledgements}

This study on hiN cell reprogramming was supported by four funds: The 2013 Project of Six Peak Talents in Jiangsu Province (grant no. WSN-022), the Project of 333 Key Talents of Science and Technology in Jiangsu Province (grant no. BRA2012094), the Scientific Project of Traditional Chinese Medicine of Jiangsu Province (grant no. LZ13195) and the 2011 Project of Scientific Technology and Social Development in Yangzhou (grant no. YZ2011084).

\section{References}

1. Davis RL, Weintraub $\mathrm{H}$ and Lassar AB: Expression of a single transfected cDNA converts fibroblasts to myoblasts. Cell 51: 987-1000, 1987.

2. Takahashi K and Yamanaka S: Induction of pluripotent stem cells from mouse embryonic and adult fibroblast cultures by defined factors. Cell 126: 663-676, 2006.

3. Yamanaka S: Pluripotency and nuclear reprogramming. Philos Trans R Soc Lond B Biol Sci 363: 2079-2087, 2008

4. Gu G, Dubauskaite J and Melton DA: Direct evidence for the pancreatic lineage: NGN3+ cells are islet progenitors and are distinct from duct progenitors. Development 129: 2447-2457, 2002.

5. Zhou Q, Brown J, Kanarek A, Rajagopal J and Melton DA: In vivo reprogramming of adult pancreatic exocrine cells to beta-cells. Nature 455: 627-632, 2008.

6. Puligilla C, Dabdoub A, Brenowitz SD and Kelley MW: Sox2 induces neuronal formation in the developing mammalian cochlea. J Neurosci 30: 714-722, 2010.

7. Liu X, Li F, Stubblefield EA, Blanchard B, Richards TL, Larson GA, He Y, Huang Q, Tan AC, Zhang D, et al: Direct reprogramming of human fibroblasts into dopaminergic neuron-like cells. Cell Res 22: 321-332, 2012.

8. Vierbuchen T, Ostermeier A, Pang ZP, Kokubu Y, Südhof TC and Wernig M: Direct conversion of firoblasts to functional neurons by defied factors. Nature 463: 1035-1041, 2010

9. Pang ZP, Yang N, Vierbuchen T, Ostermeier A, Fuentes DR, Yang TQ, Citri A, Sebastiano V, Marro S, Südhof TC and Wernig M: Induction of human neuronal cells by defined transcription factors. Nature 476: 220-223, 2011.

10. Caiazzo M, Dell'Anno MT, Dvoretskova E, Lazarevic D, Taverna S, Leo D, Sotnikova TD, Menegon A, Roncaglia P, Colciago G, et al: Direct generation of functional dopaminergic neurons from mouse and human firoblasts. Nature 476: 224-227, 2011.

11. Pfisterer U, Kirkeby A, Torper O, Wood J, Nelander J, Dufour A, Björklund A, Lindvall O, Jakobsson J and Parmar M: Direct conversion of human firoblasts to dopaminergic neurons. Proc Natl Acad Sci USA 108: 10343-10348, 2011.

12. Son EY, Ichida JK, Wainger BJ, Toma JS, Rafuse VF, Woolf CJ and Eggan K: Conversion of mouse and human fibroblasts into functional spinal motor neurons. Cell Stem Cell 9: 205-218, 2011.

13. Castro DS, Martynoga B, Parras C, Ramesh V, Pacary E, Johnston C, Drechsel D, Lebel-Potter M, Garcia LG, Hunt C, et al: A novel function of the proneural factor Ascll in progenitor proliferation identified by genome-wide characterization of its targets. Genes Dev 25: 930-945, 2011.

14. Thomas JL, Baker K, Han J, Calvo C, Nurmi H, Eichmann AC and Alitalo K: Interactions between VEGFR and notch signaling pathways in endothelial and neural cells. Cell Mol Life Sci 70: 1779-1792, 2013.

15. Hsieh FY, Ma TL, Shih HY, Lin SJ, Huang CW, Wang HY and Cheng YC: Dner inhibits neural progenitor proliferation and induces neuronal and glial differentiation in zebrafish. Dev Biol 375: 1-12, 2013.

16. Zabierowski SE, Baubet V, Himes B, Li L, Fukunaga-Kalabis M, Patel S, McDaid R, Guerra M, Gimotty P, Dahmane N and Herlyn M: Direct reprogramming of melanocytes to neural crest stem-like cells by one defined factor. Stem Cells 29: 1752-1762, 2011

17. Kutner RH, Zhang XY and Reiser J: Production, concentration and titration of pseudotyped HIV-1-based lentiviral vectors. Nat Protoc 4: 495-505, 2009. 
18. Benraiss A, Chmielnicki E, Lerner K, Roh D and Goldman SA Adenoviral brain-derived neurotrophic factor induces both neostriatal and olfactory neuronal recruitment from endogenous progenitor cells in the adult forebrain. J Neurosci 21: 6718-6731, 2011.

19. Pencea V, Bingaman KD, Wiegand SJ and Luskin MB: Infusion of brain-derived neurotrophic factor into the lateral ventricle of the adult rat leads to new neurons in the parenchyma of the striatum, septum, thalamus and hypothalamus. J Neurosci 21: 6706-6717, 2011

20. Xia Y, Wang CZ, Liu J, Anastasio NC and Johnson KM: Brain-derived neurotrophicfactor preventsphencyclidine-induced apoptosis in developing brain by parallel activation of both the ERK and PI-3K/Akt pathways. Neuropharmacology 58: 330-336, 2010.

21. Mullen LM, Pak KK, Chavez E, Kondo K, Brand Y and Ryan AF: Ras/p38 and PI3K/Akt but not Mek/Erk signaling mediate BDNF-induced neurite formation on neonatal cochlear spiral ganglion explants. Brain Res. 1430: 25-34, 2012.

22. Südhof TC: The structure of the human synapsin I gene and protein. J Biol Chem 265: 7849-7852, 1990.

23. Huttner WB, Schiebler W, Greengard P and De Camilli P: Synapsin I (protein I), a nerve terminal-specific phosphoprotein. III. Its association with synaptic vesicles studied in a highly purified synaptic vesicle preparation. J Cell Biol 96: 1374-1388, 1983.

24. Osaki M, Oshimura M and Ito H: PI3K-Akt pathway: Its functions and alterations in human cancer. Apoptosis 9: 667-676, 2004.

25. Huang $S$ and Houghton PJ: Targeting mTOR signaling for cancer therapy. Curr Opin Pharmacol 3: 371-377, 2003.

26. Qian Y, Corum L, Meng Q, Blenis J, Zheng JZ, Shi X, Flynn DC and Jiang BH: PI3K induced actin filament remodeling through Akt and p70S6K1: Implication of essential role in cell migration. Am J Physiol Cell Physiol 286: C153-C163, 2004.
27. Wapinski OL, Vierbuchen T, Qu K, Lee QY, Chanda S, Fuentes DR, Giresi PG, Ng YH, Marro S, Neff NF, et al: Hierarchical mechanisms for direct reprogramming of fibroblasts to neurons. Cell 155: 621-635, 2013.

28. Bertrand N, Castro DS and Guillemot F: Proneural genes and the specification of neural cell types. Nat Rev Neurosci 3: 517-530, 2002.

29. Ross SE, Greenberg ME and Stiles CD: Basic helix-loop-helix factors in cortical development. Neuron 39: 13-25, 2003.

30. Banito A and Gil J: Induced pluripotent stem cells and senescence: Learning the biology to improve the technology. EMBO Rep 11: 353-359, 2010.

31. Salama I, Malone PS, Mihaimeed F and Jones JL: A review of the S100 proteins in cancer. Eur J Surg Oncol 34: 357-364, 2008.

32. Andrés R, Mayordomo JI,Zaballos P, Rodino J, Isla D, Escudero P, Elosegui L, Filipovich E, Saenz A, Polo E and Tres A: Prognostic value of serum S-100B in malignant melanoma. Tumori 90: 607-610, 2004

33. Blesch A, Bosserhoff AK, Apfel R, Behl C, Hessdoerfer B, Schmitt A, Jachimczak P, Lottspeich F, Buettner R and Bogdahn U: Cloning of a novel malignant melanoma-derived growth regulatory protein, MIA. Cancer Res 54: 5695-5701, 1994.

34. Kazakova MH and Sarafian VS: YKL-40-a novel biomarker in clinical practice? Folia Med (Plovdiv) 51: 5-14, 2009.

35. Yilmazer A, de Lázaro I and Taheri H: Reprogramming cancer cells: A novel approach for cancer therapy or a tool for disease-modeling? Cancer Lett 369: 1-8, 2015.

36. Cheng H, Liu C, Cai X, Lu Y, Xu Y and Yu X: iPSCs derived from malignant tumor cells: Potential application for cancer research. Curr Stem Cell Res Ther 11, 1-7, 2016.

37. Su Z, Zang T, Liu ML, Wang LL, Niu W and Zhang CL: Reprogramming the fate of human glioma cells to impede brain tumor development. Cell Death Dis 5: e1463, 2014. 